\title{
Diversidad y composición de pteridofitas en tres formaciones vegetales en el rio Los Amigos, Madre de Dios, Amazonía sur Peruana
}

\section{Diversity and composition of pteridophytes in three plant formations in the Los Amigos river, Madre de Dios, South Peruvian Amazonia}

\author{
Benjamín R. Chambi Pacompía
}

Facultad de Ciencias Agrarias de la Universidad Nacional de San Antonio Abad del Cusco (Sede Madre de Dios). Jirón San Martin Nro.451,Provincia Tambopata,Región Madre de Dios,Perú.E-mail:bchambip@gmail.com

\section{Resumen}

La composición florística, diversidad alfa y beta de pteridofitas fue estudiada en el río Los Amigos de la región Madre de Dios, Perú. Se evaluaron 12 transectos ( $5 \mathrm{~m}$ x 500m) distribuidos equitativamente y sistemáticamente en tres formaciones vegetales. Donde se registró un total 22,810 individuos de pteridofitas, distribuidos en 92 especies (se incluye 7 morfoespecies), 37 géneros y 18 familias. El bosque inundable (57 especies) posee la más alta diversidad alfa, seguido por el bosque aguajal (44) y tierra firme (38). Del mismo modo, se encontró elevada diversidad beta entre las formaciones vegetales. Se confirma que la variación ambiental influye sobre diversidad beta de las pteridofitas y pueden ser usados para la clasificación de la vegetación.

Palabras claves: Amazonía peruana, diversidad alfa y beta, Pteridofitas, Río Los Amigos.

\begin{abstract}
The floristic composition, alpha and beta diversity of pteridophytes was studied in the Los Amigos river in the Madre de Dios region, Peru. Twelve transects (5m x 500m) distributed equally and systematically in three plant formations were evaluated. Where a total of 22,810 individuals of pteridophytes were registered, distributed in 92 species (including 7 morpho species), 37 genera and 18 families. The flood plain forest ( 57 species) has the highest alpha diversity, followed by the wetland forest (44) and terra firme (38). Similarly, high beta diversity was found among plant formations. It is confirmed that environmental variation influences the beta diversity of pteridophytes and can be used for the classification of vegetation.
\end{abstract}

Keyword: peruvian Amazon, diversity alpha and beta, Pteridofitas, river Los Amigos. 


\section{Introducción}

Las diversidad, riqueza y composición de especies de pteridofitas se han estudiado desde varios años atrás en los bosques del neotrópico (Young \& León 1989, Araujo 1999, Tuomisto \& Poulsen 2000, Kessler 2002, Santiago et al. 2004, Rodríguez et al. 2004, Dittrich et al. 2005, Cárdenas et al. 2007, Young 2007, SoriaAuza 2009, Cassá de Pazos et al. 2010, Mondragón \& García 2011, Martínez \& Prado 2013).

La composición de especies de helechos y las familias Melastomataceae y Myristicaceae pueden usarse para la clasificación de tipos de bosques y patrones Fitogeográficos a gran escala en la Amazonía (Ruokolainen et al. 1994 y 1997, Salovaara et al. 2004), con esos grupos de plantas, hierbas angiospermas terrestres y palmeras se puede conocer el grado de la especialización edáfica, las cuales son suficientes para justificar el uso como especies indicadores de la fertilidad de los suelos (Tuomisto \& Poulsen 1996, Tuomisto et al. 2002. Cárdenas et al. 2003) y, son importantes para la distribución y diversidad beta de plantas a una escala local (Tuomisto et al. 1998, Poulsen et al. 2006, Tuomisto \& Ruokolainen 2005, Cárdenas et al. 2007, Zuquim et al. 2009). Sin embargo, no son confiables para predecir patrones en la diversidad alfa (Tuomisto \& Ruokolainen 2005). Estos trabajos confirman que dentro de una determinada zona climática, diversidad de tipos de suelo y la posición topográfica pueden coexistir tantas especies de pteridofitas especialistas edáficos y generalistas (Young \& León 1989), además se pueden conocer las diferencias funcionales en la ecología de helechos epifitas y terrestres (James et al. 2009).
Por otro lado, los patrones de abundancia de Pteridofitas y Melastomataceae están asociadas a la posición topográfica (Tuomisto \& Poulsen 2000, Poulsen et al. 2006) se relacionaron con las imágenes de satélite para entender los patrones de diversidad de grupos de plantas o patrones fitogeográficos (Tuomisto 1998a, Tuomisto et al. 2003 b, c). No obstante, la riqueza y distribución de Pteridofitas en fragmentos revelan el impacto sobre la diversidad de especies de flora, principalmente en el borde del bosques (de Granville 1984, Beukema \& Noordwijk 2004, Rodríguez et al. 2004, Williams-Linera et al. 2005, Rodríguez et al.2008), y que las distintas características del ambiente afectan sobre su morfofisiología (Gómez 2012), posibles argumentos para considerar su conservación. También dentro de los bosques primarios en la reserva de producción faunística de la amazónica de Cuyabeno de Ecuador, se estudió la riqueza de especies de pteridofitas dentro de 1 ha (Poulsen \& Nielsen 1995), y de similar manera se hizo en Singapur y Malasia hicieron intentos por determinar el área mínima de muestreo para estimar la biodiversidad de pteridofitas en ambientes naturales y artificiales (Yusuf et al.2003).

En bosques de Madre de Dios, se realizaron pocos estudios sobre la diversidad y composición de Pteridofitas (Rodríguez et al. 2003, Janovec et al. 2013), y se documentó poco sobre el uso como indicadores de patrones de diversidad en relación con la concentración de cationes del suelo (Tuomisto \& Ruokolainen 2005).

En el presente estudio, evaluamos a través de transectos tres formaciones vegetales (a. Bosque Tierra firme, b. Inundables y c. Aguajal), donde registramos la diversidad y- 
-composición de especies, estos datos nos permitió determinar la diversidad alfa y beta en la zona de la desembocadura del río Los Amigos, Madre de Dios. Los objetivos del estudio fueron: a) Evaluar la composición de especies de pteridofitas en las tres formaciones vegetales y b) Analizar la diversidad alfa y beta de cada una y entre tipos de bosques.

\section{Materiales y métodos}

\section{Área de estudio}

La investigación se realizó en un zona donde confluyen el área del Centro de Investigación y Capacitación Río Los Amigos (CICRA), Concesión de Conservación Los Amigos (CCLA) y una pequeña área de una concesión maderera al norte del CICRA, estas se ubican en la provincia de Manu de la región Madre de Dios, Perú y en un altitud desde los 140 hasta $290 \mathrm{msnm}$ aproximadamente (Figura 1). Las 3 formaciones vegetales están en bosques de orillas o llanuras meándricas tienen suelos limo-arcillosos a arcillosos (Encarnación et al. 2008). Según Holdridge (1978) se ubica en la zona bosque húmedo tropical. Geomorfológicamente se caracteriza; planicie aluvial y Terrazas recientes y sub-recientes a nivel de gran paisaje respectivamente, están conformadas por el complejo de orillares, terrazas altas disectadas y bajas, con depósitos aluviales recientes y sub-recientes (Encarnación et al.2008).

El bosque de terraza alta (UTM 19 L 380130E, 8611900N) de Madre de Dios presenta suelos con textura arenosa (66.6\%), limosos (21\%) y arcillosos (12\%) (García, 2005) y son pobres en nutrientes y ácidos (Pitman 2000), pero estas propiedades edáficas pueden variar a gran escala (Mezer 1997), su composición florística está conformada por
- por familias dominantes Fabaceae, Moraceae, Malvaceae, Lecythidaceae, Arecaceae entre otras (Pitman et al.2001), con un dosel de aproximadamente $40 \mathrm{~m}$ de altura y con abundantes epifitas.

Los bosques de terrazas inundables (UTM 19 L 381755E, 8610100N) presentan suelos con texturas francas, ocurre inundaciones temporales en épocas de lluvia, dominado por árboles dominantes y emergentes en las familias Moraceae, Malvaceae, Fabaceae, Sapotaceae, Arecaceae, que sirve como forófitos para pteridofitas, orquídeas y aráceas. El bosque Aguajal (UTM 19 L 378284E, $8611394 \mathrm{~N}$ ) se sitúa en superficie depresionado, presenta suelos arcillosos y arcilla-arenoso, y con drenaje pobre, saturado con agua de coloración negra por la destilación de taninos y abundante materia orgánica producida principalmente por troncos y hojas de palmeras (Householder 2007, Kalliola et al. 1991, Janusz 2002). Su vegetación es dominado por aguaje (Mauritia flexuosa, Fam. Arecaceae), seguida por especies de Hedyosmum (Chloranthaceae), Ilex (Aquifoliaceae), Tapirira (Anacardiaceae) Tabebuia (Bignoniaceae), Lueheopsis (Malvaceae), Ficus (Moraceae) entre otras, estas sirven como hospederos para las plantas epifitas como la Vanilla (fam. Orchidaceae) (Janovec et al. 2013).

\section{Registro de Pteridofitas}

Se evaluaron 12 transectos de $5 \mathrm{~m}$ x 500m, distribuidos cuatro transectos en cada formación vegetal (similar a Tuomisto et al. 2002; Toumisto \& Ruokolainen 2005). Donde registramos todas las pteridofitas terrestres, acuáticas, epifitas y hemiepifitas, estos dos últimos en transectos, solo hasta los $2 \mathrm{~m}$ de altura de los forófitos. 


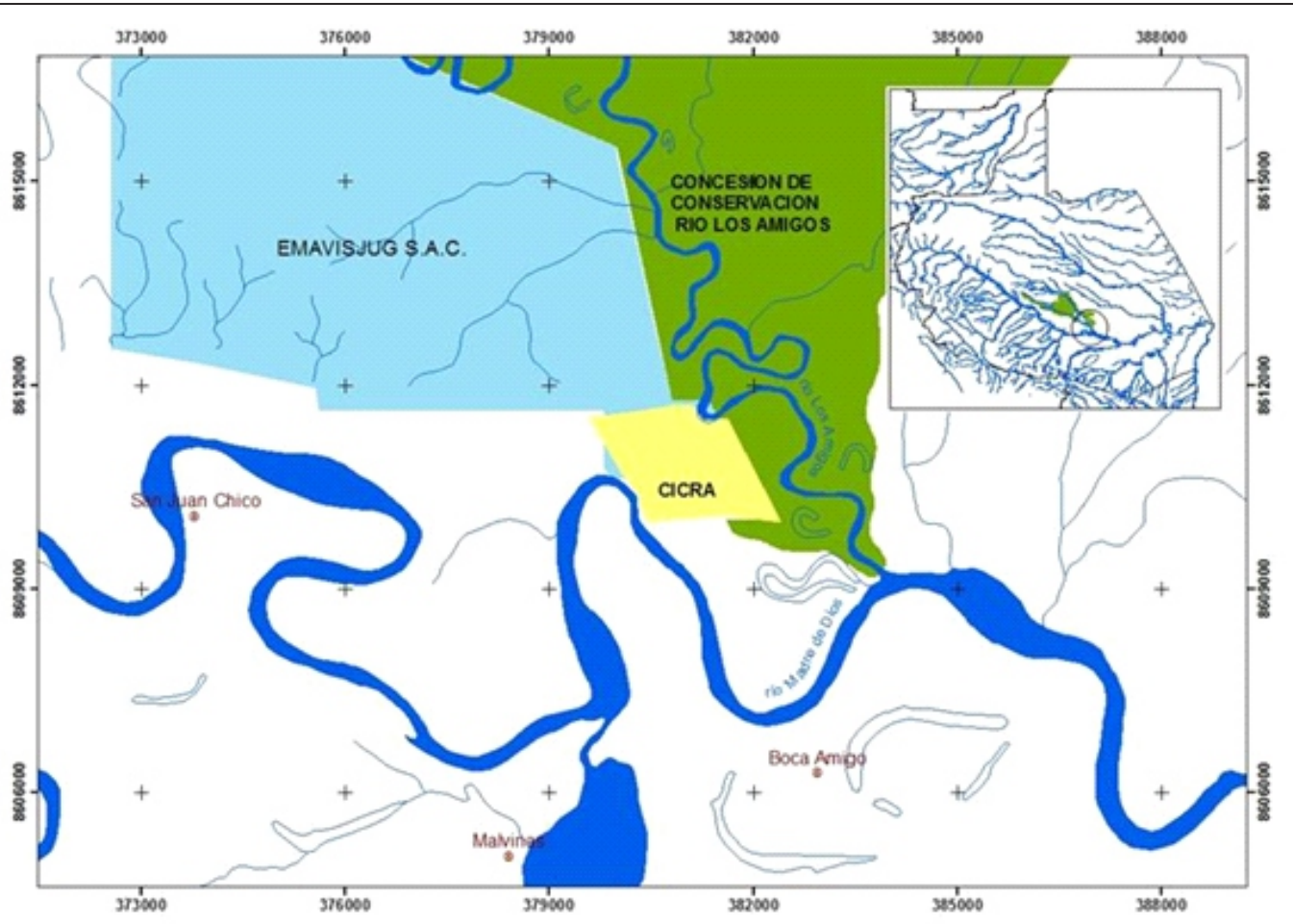

Figura 1. Ubicación del área de estudio en el sector río Los Amigos, región Madre de Dios, Perú.

Las identificaciones taxonómicas preliminares en campo se realizaron con claves taxonómicas en Tryon \& Stolze (1989, 1991a, 1991b, 1994) y el taxón de cada especies de pteridofita fueron confirmadas por especialistas en este grupo de plantas. Las muestras botánicas fueron depositadas en el Herbario del Instituto de Investigación Botánica de Texas (BRIT) de Estados Unidos y Herbario San Marcos de la Universidad Nacional de San Marcos (USM).

\section{Análisis de datos}

Para conocer la diversidad alfa de las tres formaciones vegetales utilizamos la riqueza de especies (S) y el Índice de Shannon Wiener $(\mathrm{H})$ : $\mathrm{H}=-\sum \mathrm{p}_{\mathrm{i}} \ln \mathrm{p}_{\mathrm{i}}$. Donde $\mathrm{p}_{\mathrm{i}}$ es la abundancia proporcional de una especie (Tuomisto 2010)- -y Margalef $(\mathrm{DMg}=\mathrm{S}-1 / \mathrm{Ln} \mathrm{N}$; Donde: $\mathrm{S}=$ número de especies, $\mathrm{N}=$ número total de individuos).

Del mismo modo, para determinar el grado de reemplazo de especies o cambio biótico a través de gradientes ambientales (Moreno 2001; Tuomisto 2010) se utilizó el coeficiente de Jaccard: $I_{J}=C / a+b-c$, Donde: $\mathrm{a}=$ número de especies presentes en el sitio $\mathrm{A}, \mathrm{b}=$ número de especies presentes en el sitio $\mathrm{B}, \mathrm{c}=$ número de especies presentes en ambos sitios A y B.

Las curvas de acumulación de especies se realizó con el programa EstimateS v7.5.0, con el cual se determinó el índice de Mao Tao para cada subparcela $5 \mathrm{~m}$ x 100m. 


\section{Análisis Multivariado}

Para conocer los patrones de diversidad de pteridofitas en cada formación vegetal, se elaboró una matriz constituida por especies y abundancia de pteridofitas distribuidas en subparcelas ( $5 \mathrm{~m}$ x $100 \mathrm{~m}$ ), a la cual se aplicó el Análisis de Principales Coordenadas, con el Método de unión por promedio aritmético de grupos de pares no ponderados (UPGMA) asociado al Índice de Jaccard. A esa matriz se hizo la conversión lineal en dos ejes, para ello se utilizó el índice de Bray-Curtis, este permitió ver el patrón espacial y de diversidad, ambos fueron computados por Multi-Variate Statistical Package v3.1 (Kovach Computing Services 2007).

\section{Resultados}

\section{Riqueza y composición de especies}

Se cuantificaron 22,810 individuos de pteridofitas, representado por un total de 92 especies (se incluye 7 morfoespecies), 37 géneros y 18 familias (Tab. 1.). Estas presentan las siguientes formas de vida; terrestres (55 especies), epifitas (25), hemiepífitas (11) y acuáticas (2). La riqueza de especies más alta fue registrada para el bosque inundable (57 especies), seguido por el B. aguajal (44) y en menor proporción en el bosque de tierra alta o tierra firme (38). En el bosque de terraza baja (estacionalmente inundable) vario de 20 a 34 especies por transecto, mientras que en el bosque aguajal (22 a 31) y terraza alta (18 a 30) tuvieron menor cantidad de riqueza de especies (Tabla 2).

Asimismo, las familias compuestas con elevado número de especies son Polypodiaceae (13), Pteridaceae (10), Hymenophyllaceae (9), Thelypteridaceae (9) y Lomariopsidaceae-
-(7). Catorce de las 18 familias están en el bosque aguajal e inundable, mientras que 13 solo están en el bosque de tierra firme.

Asimismo, al analizar la acumulación de especies a nivel de subparcelas, se puede observar en la Figura 2, que los bosques de terraza alta y terraza baja presentan curvas de acumulación de especies con tendencia a formar una asíntota, mientras que el bosque aguajal aún continúa en ascendencia. Esto indica que es necesario incrementar el esfuerzo de muestreo para documentar la totalidad de especies de pteridofitas en los tres sitios evaluados.

\section{Abundancia}

El bosque aguajal (14,493 individuos) presenta mayor abundancia que las otras dos formaciones vegetales. Este bosque está dominada por especies según orden decreciente como: Nephrolepis biserrata (11.1\% de la abundancia total), Cyclodium miniscioides, Selaginella lepidophylla (8.2\%), Polybotrya caudata (6.3\%), Cyathea pungens (5.6\%), Trichomanes cristatum (5.1\%) y Polypodium adnatum (4.5\%). En el bosque inundable (5,393 individuos): Polybotrya caudata (6.1\%), Adiantum humile (5.1\%), Lomariopsis japurensis (3.8\%), Bolbitis lindigii (1.9\%), Bolbitis nicotianifolia $(1.8 \%)$ y Adiantum aff. petiolatum (1.3\%). Y en bosque de tierra firme $(2,924$ individuos) destacan: Lomariopsis japurensis (2.9\%), Diplazium gandifolium (2.1\%), Adiantum tetraphyllum (1.4\%), Polybotrya pubens (1.3\%) y Lomagramma guianensis (1.1\%).

Del mismo modo, las familias con mayor abundancia en los tres tipos de bosques son Dryopteridaceae (22.5\%), Lomariopsidaceae (13.9\%), Davalliaceae (1 $1.1 \%)$, Selaginellaceae (8.6\%). Paradójicamente, la mayoría de estas, no están entre las más ricas - 


\begin{tabular}{|c|c|c|c|c|}
\hline Familia y especie & A & B & $\mathbf{F}$ & $\begin{array}{c}\text { Nro. } \\
\text { Individuos }\end{array}$ \\
\hline \multicolumn{5}{|l|}{ Aspleniaceae } \\
\hline Asplenium cirrhatum Rich. ex Willd. & 17 & 21 & 167 & 205 \\
\hline Asplenium cuspidatum Lam. & & 30 & & 30 \\
\hline Asplenium pearcei Baker & & 11 & 2 & 13 \\
\hline Asplenium serratum L. & 2 & 45 & 13 & 60 \\
\hline Asplenium stuebelianum Hieron. & & 1 & 2 & 3 \\
\hline \multicolumn{5}{|l|}{ Cyatheaceae } \\
\hline Alsophila cuspidata (Kunze) D.S. & & 9 & & 9 \\
\hline Cnemidaria horrida (L.) C. Presl. & & 4 & 3 & 7 \\
\hline Cyathea pungens (Willd.) & 1272 & 30 & 3 & 1305 \\
\hline Cyathea sp brc140 & & 1 & 122 & 123 \\
\hline \multicolumn{5}{|l|}{ Davalliaceae } \\
\hline Nephrolepis biserrata (Sw.) Schott & 2531 & & & 2531 \\
\hline Nephrolepis rivularis (Vahl) Mett. ex Krug & 10 & & & 10 \\
\hline \multicolumn{5}{|l|}{ Dennstaedtiaceae } \\
\hline Dennstaedtia bipinnata (Cav.)Maxon. & & 7 & & 7 \\
\hline Lindsaea divaricata Klotzsch & 5 & & & 5 \\
\hline Lindsaea quadrangularis Raddi. & 900 & & & 900 \\
\hline Lindsaea stricta (Sw.) Dryand. & 158 & & & 158 \\
\hline Lindsaea taeniata K.U. Kramer & & & 4 & 4 \\
\hline Saccoloma inaequale (Kunze) Mett. & 2 & 20 & 68 & 90 \\
\hline \multicolumn{5}{|l|}{ Dryopteridaceae } \\
\hline Ctenitis sloanie (Poepp. ex Spreng.)C.V.Morton. & & 1 & & 1 \\
\hline Cyclodium meniscioides (Willd.) C. Presl & 1874 & & & 1874 \\
\hline Polybotrya caudata Kunze & 1448 & 1382 & 86 & 2916 \\
\hline Polybotrya crassirhizzoma Lellinger & & 16 & 28 & 44 \\
\hline Polybotrya pubens Mart. & & & 304 & 304 \\
\hline Stigmatopteris heterophlebia (Baker) R.C. Moran & & 2 & & 2 \\
\hline \multicolumn{5}{|l|}{ Hymenophyllaceae } \\
\hline Trchomanes sp brc170 & 1 & & & 1 \\
\hline Trichomanes cf. Trollii & & & 5 & 5 \\
\hline Trichomanes collariatum Bosch & & 21 & & 21 \\
\hline Trichomanes cristatum Kaulf. & 1156 & & & 1156 \\
\hline Trichomanes elegans Rich. & & & 8 & 8 \\
\hline Trichomanes martiusii C. Presl & 574 & & & 574 \\
\hline Trichomanes reptans $\mathrm{Sw}$. & 1 & 3 & & 4 \\
\hline Trichomanes sphenoides Kunze & 1 & 10 & & 11 \\
\hline Tricomanes pinnatum Hedw. & 17 & & 28 & 45 \\
\hline \multicolumn{5}{|l|}{ Isoëtaceae } \\
\hline Isoëtes panamensis Maxon \& C.V. Morton & 117 & & & 117 \\
\hline \multicolumn{5}{|l|}{ Lomariopsidaceae } \\
\hline Bolbitis lindigii (Mett.) C. Chr. & & 430 & & 430 \\
\hline Bolbitis nicotianifolia $\left(S_{w}\right)$ Alston & & 407 & & 407 \\
\hline Elaphoglossum flaccidum (Fée) T. Moore & 1 & & & 1 Continua... \\
\hline
\end{tabular}




\section{Marattiaceae}

Danaea elliptica Sm.

Danaea leprieurii Kunze

Danaea nodosa (L.) Sm.

$\begin{array}{ccc} & 3 & \\ 2 & 96 & 98 \\ 37 & 13 & 25\end{array}$

Metaxyaceae

Metaxia rostrata (Humb. \& Bonpl. ex Willd.) C.Presl.

\section{Polypodiaceae}

Campyloneurum fuscosquamatum Lellinger

$11 \quad 51 \quad 62$

Campyloneurum phyllitidis (L.) C. Presl

$\begin{array}{llll}30 & 36 & 11 & 77\end{array}$

Microgramma percussa (Cav.) de la Sota

Microgramma persicariifolia (Schrad.) C. Presl

Microgramma reptans (Cav.) A.R. Sm.

$3 \quad 21$

Microgramma tecta (Kaulf.) Alston

Microgramma thurnii (Baker) R.M. Tryon \& Stolze

1

Pecluma aff. plumula

$\begin{array}{llll}25 & 18 & 1 & 44\end{array}$

2

23

Pecluma plumula (Humb. \& Bonpl. ex Willd.) M.G. Price

Pleopeltis fuscopunctata (Hook.) R.M. Tryon \& A.F. Tryon

$$
\begin{aligned}
& 98 \\
& 25
\end{aligned}
$$

Polypodium adnatum Kunze ex Klotzsch

Polypodium bombycinum Maxon

Polypodium triseriale Sw.

\section{Pteridaceae}

Adiantum aff. petiolatum

Adiantum dolosum Kunze

$\begin{array}{cccc} & 286 & & 286 \\ & 1 & 13 & 14 \\ & 1170 & & 1170 \\ & & 2 & 2 \\ & 10 & & 10 \\ & & 20 & 20 \\ 5 & 2 & 311 & 318 \\ & 6 & & 6 \\ & 5 & & 5 \\ & 5 & & 5 \\ & & & 128 \\ 128 & & & 186 \\ & & & 13 \\ 15 & 72 & & \end{array}$

Adiantum humile Kunze

Adiantum obliquum Willd.

Adiantum pulverulentum $\mathrm{L}$.

Adiantum terminatum Kunze ex Miq.

Adiantum tetraphyllum Humb. \& Bonpl. ex Will d.

Adiantum tomentosum Klotzsch

Pteris propinqua J. Agardh

Pteris pungens Willd.

Schizaeaceae

Schizaea poeppigiana J.W. Sturm

\section{Selaginellaceae}

Selaginella exalta (Kunze) Spring.

Selaginella lepidophylla (Hook. \& Grev.) Spring

\section{Tectariaceae}

Tectaria brauniana (H. Karst.) C. Chr.

Tectaria draconoptera (D.C. Eaton) Copel.

Tectaria incisa Cav. 
Continuación

Triplophyllum sp. bcp13

\section{Thelypteridaceae}

Telypteris sp brc107

$\begin{array}{ccc} & 49 & 49 \\ & 4 & 4 \\ 229 & & 229 \\ 1 & 11 & 12 \\ & 33 & 33 \\ & 33 & 33 \\ 2 & & 2 \\ 154 & & 154 \\ & 52 & 52\end{array}$

Thelypteris ancyriothrix (Rosenst.) A.R. Sm.

49

Thelypteris sp brc82

Thelypteris jamesonii (Hook.) R.M. Tryon

Thelypteris juruensis (C. Chr.) R.M. Tryon \& D.S. Conant

Thelypteris sp brc 80

Thelypteris sp. brc20

Thelypteris sp. brc22

Thelypteris toganetra A.R. Sm.

Vittariaceae

Ananthocorus angustifolia (Sw.) Underw. \& Maxon

Anetium citrifolium (L.) Splitg.

$3 \quad 3 \quad 6$

Hecistopteris pumila (Spreng.) J. Sm.

$\begin{array}{llll}8 & 3 & 7 & 18\end{array}$

Polytaenum cajenense (Desv.) Benedict

Polytaenun guayanense (Hieron.) Alston

Woodsiaceae

Diplazium grandifolium (Sw.) Sw.

Diplazium sp brc150

$14493 \quad 5393 \quad 2924$

22810

Tabla 2. Abundancia, riqueza y diversidad de especies de pteridofitas encontrados en tres formaciones vegetales en Los Amigos, Madre de Dios, SE de la Amazonía peruana.

\begin{tabular}{|c|c|c|c|c|c|c|c|c|c|c|c|c|c|c|c|}
\hline Parámetr os & A1 & A2 & A3 & A4 & $\begin{array}{c}\text { Total } \\
\text { A }\end{array}$ & B1 & B2 & B3 & B4 & $\begin{array}{c}\text { Total } \\
\text { B }\end{array}$ & F1 & F2 & F3 & $\mathbf{F} 4$ & $\begin{array}{c}\text { Total } \\
\text { F }\end{array}$ \\
\hline Taxa (S) & 29 & 22 & 24 & 31 & 44 & 28 & 20 & 26 & 34 & 57 & 30 & 19 & 26 & 18 & 38 \\
\hline Abundancia & 5388 & 3505 & 2419 & 3181 & 14493 & 1554 & 442 & 1704 & 1693 & 5393 & 1169 & 315 & 742 & 698 & 2924 \\
\hline Shannon $\mathrm{H}^{\prime}$ & 2.34 & 2.29 & 2.19 & 2.35 & 2.53 & 1.54 & 2.17 & 1.94 & 2.37 & 2.35 & 2.58 & 1.91 & 2.25 & 2.03 & 2.56 \\
\hline Margalef & 3.26 & 2.57 & 2.95 & 3.72 & 4.49 & 3.67 & 3.12 & 3.36 & 4.44 & 6.52 & 4.11 & 3.13 & 3.78 & 2.6 & 4.64 \\
\hline
\end{tabular}

- en especies, a excepción de Lomariopsidaceae, que ocupa el quinto lugar en un orden jerárquico. De las 4 familias con alta abundancia para las 3 formaciones vegetales, Lomariopsidaceae no está entre las más abundantes para el bosque aguajal, sin embargo Hymenophyllaceae (7.7\%) se adiciona para este hábitat. También Dryopteridaceae $(6.1 \%, 1.8 \%)$ -
Lomariopsidaceae $(7.7 \%, 4 \%)$ y Pteridaceae $(6.5 \%, 1.5 \%)$ son las más abundantes en los bosques terraza baja y alta. A este último bosque se adiciona la familia Woodsiaceae (2.1\%).

Los patrones de abundancia vario desde 2,419 a 5,388 individuos de pteridofitas por transecto, mientras que el bosque de terraza baja (442 a $1,704)$ y alta $(315$ a 1,169$)$ tuvieron menos abundancia en sus transectos (Tabla 2). 


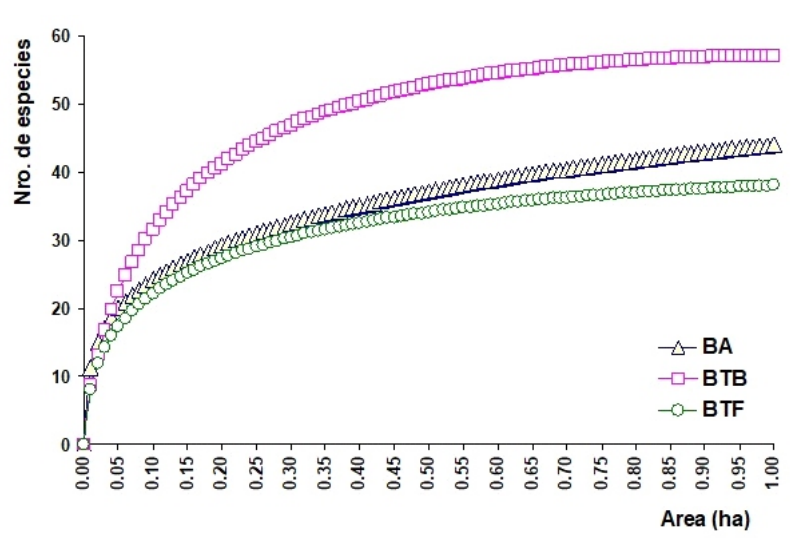

Figura 2. Según el Índice de MaoTao, se muestra la acumulación de especies de pteridofitas en tres formaciones vegetales: $\mathrm{BA}=$ Bosque Aguajal, $\mathrm{BTB}=$ Bosque de terraza baja y $\mathrm{BTA}=$ Bosque de terraza alta.

\section{Patrones de diversidad}

Según el índice de Margalef $\left(M_{g}\right)$ la diversidad de pteridofitas más alta fue registrada en el bosque inundable $\left(M_{g}=6.52\right)$, y en menor proporción en el bosque de Tierra firme $\left(M_{g}=4.64\right)$ y Aguajal $\left(M_{g}=4.49\right)$. Sin embargo, el índice de Shannon (H) determina que el bosque de tierra firme es el más diverso, y menos diverso bosque aguajal y bosque de terraza baja. Estos índices están influidos por la abundancia de individuos, y estos no coinciden con la riqueza de especies de pteridofitas por formación vegetal (Tabla 3).

Por otro lado, según el coeficiente de Jaccard (presencia-ausencia), entre el bosque aguajal y terraza alta presentan la más baja similitud en composición de especies $(J=0.24)$, y la más alta $(J=0.30)$ cuando comparamos entre el bosque de terraza alta y bosque de terraza baja. Al comparar el bosque de terraza baja con bosque aguajal presenta similitud intermedia $(J$ $=0.28)$. El grado de reemplazo de especies entre las tres formaciones vegetales, es alto- menos del 30\% de especies comparten entre sitios evaluados. En contraste, al comparar entre transectos de un tipo de bosque, el bosque aguajal presenta altas similitudes de especies entre el A3 y A2 (70\%) y entre A3 y A4 (62\% comparten especies), mientras que A1 difiere altamente con los demás transectos.

Similarmente, al comparar los transectos entre F1 y F3 (60\%), F1 y F4 (55\%) y F3 y F4 (57\%) comparten más del 50\% de las especies presente en el bosque de terraza alta. Este patrón de similitud, es menor al comparar la composición de especies entre los transectos del bosque de terraza baja (Tabla 3).

Tabla 3. Matriz de similaridad de especies de pteridofitas de transecto dentro y entre tipos de bosques.

\begin{tabular}{|c|c|c|c|c|c|c|c|c|c|c|c|}
\hline & A1 & A2 & A3 & A4 & B1 & B2 & B3 & B4 & F1 & F2 & \multirow[t]{2}{*}{3} \\
\hline $\mathrm{A} 1$ & 1 & & & & & & & & & & \\
\hline A2 & 0.417 & 1 & & & & & & & & & \\
\hline $\mathrm{A} 3$ & 0.395 & \begin{tabular}{ll|}
0.704 \\
\end{tabular} & 1 & & & & & & & & \\
\hline A4 & 0.463 & 0.606 & 0.618 & 1 & & & & & & & \\
\hline B1 & 0.096 & 0.163 & 0.238 & 0.157 & 1 & & & & & & \\
\hline B2 & 0.114 & 0.077 & 0.222 & 0.133 & 0.455 & 1 & & & & & \\
\hline B3 & 0.058 & 0.091 & 0.163 & 0.118 & 0.459 & 0.438 & 1 & & & & \\
\hline B4 & 0.123 & 0.163 & 0.18 & 0.2 & 0.313 & 0.25 & 0.298 & 1 & & & \\
\hline $\mathrm{F} 1$ & 0.113 & 0.238 & 0.227 & 0.245 & 0.184 & 0.087 & 0.098 & 0.275 & 1 & & \\
\hline $\mathrm{F} 2$ & 0.116 & 0.206 & 0.194 & 0.22 & 0.146 & 0.114 & 0.098 & 0.174 & 0.441 & 1 & \\
\hline F3 & 0.146 & 0.263 & 0.22 & 0.267 & 0.2 & 0.07 & 0.106 & $0.245^{\circ}$ & \begin{tabular}{l|l}
0.6 \\
\end{tabular} & 0.5 & 1 \\
\hline $\mathrm{F} 4$ & 0.146 & 0.29 & 0.273 & 0.289 & 0.15 & 0.086 & 0.073 & 0.293 & 0.548 & 0.542 & 0.571 \\
\hline
\end{tabular}

\section{Patrones de distribución}

En total 13 especies de pteridofitas se distribuyen en los tres tipos de bosques. Las especies con alta abundancia según el orden decreciente son Polybotrya caudata, Lomariopsis japurensis, Cyathea pungens, Lomagramma guianensis y Adiantum tetraphyllum. Y entre las especies terrestres destacan; Adiantum tetraphyllum, Asplenium cirrhatum y Saccoloma inaequale. Están presentes en orilla del aguajal, donde es baja la profundidad de materia orgánica. 
Sin embargo, en el bosque aguajal se encontró especies con más alta abundancia; Cyathea pungens, Lomagramma guianensis, Poybotrya caudata, Microgramma reptans, y en menor proporción se distribuye en el bosque terraza baja (inundable) y terraza alta. En este último hábitat, en micrositios con alta humedad. También en el bosque aguajal, las especies con forma de vida epífitas y hemiepífitas como Lomagramma guianenesis, Lomariopsis japurensis, Asplenium cirrhatum, Asplenium serratum, Campyloneurum phyllitidis y Anetium citifolium encontramos sobre tallos de palmeras y árboles del aguajal cercano a su orilla, mientras que los otros dos tipos de bosques, están dispersos aleatoriamente.

No obstante, en el bosque de terraza baja destacan especies como Lomariopsis japurensis, Asplenium serratum y Campyloneurum phyllitidis, y estas con menor abundancia en el bosque de terraza alta y aguajal. En el bosque de terraza alta destacan especies como Adiantum tetraphyllum, Asplenium cirrhatum, Pleopeltis fuscopunctata y Saccoloma inaequale, y están presentes en menor cantidad de individuos en los otros bosques citados.

Al comparar la distribución de especies de pteridofitas entre el bosque aguajal y terraza alta solo comparten el 23\%, y estos bosques al comparar con el bosque de terraza baja solo comparten el 15\% de la flora pteridológica (Figura 3).

Del total de especies de pteridofitas registradas para el área de estudio, 27 especies son exclusivas para el bosque de terraza baja, seguido 19 para el bosque de aguajal y 13 para el bosque de terraza alta (Fig. 3). Estas especies restringidas para el bosque de terraza alta destacan; Adiantum tetraphyllum, Bolbitis lindigii,
- Bolbitis nicotianifolia y Adiantum aff. petiolatum, en el bosque aguajal; Nephrolepis biserrata, Cyclodium miniscioides, Selaginella lepidophylla, Trichomanes cristatum, Polypodium adnatum y Lindsaea quadrangularis. Y en bosque de terraza alta; Polybotrya pubens, Metaxia rostrata y Triplophyllum funestum, Nephrolepis biserrata, Cyclodium miniscioides, Selaginella lepidophylla, Trichomanes cristatum, Polypodium adnatum y Lindsaea quadrangularis. Y en bosque de terraza alta; Polybotrya pubens, Metaxia rostrata y Triplophyllum funestum.

Al analizar en subparcelas de $500 \mathrm{~m}^{2}$ (Figura 4), estas se diferencian entre las tres formaciones vegetales. El conjunto de subparcelas del bosque aguajal presentan un gradiente en el cambio en composición de especies de pteridofitas, cuyas subparcelas cercanas al bosque de terraza alta tienden a agruparse a este. En contraste, las subparcelas del bosque de terraza alta tienen alta similaridad en especies y tienden agruparse entre sí. No obstante, a pesar que el bosque de terraza baja es el más diverso y con alto número de especies exclusivas, comparte 22 especies con el bosque de terraza alta y otras 22 con el bosque aguajal. Esta particularidad muestra un conjunto de subparcelas sutilmente dispersas, algunas de ellas con tendencia a agruparse a los otros dos bosques evaluados.

Cabe indicar, que en el centro del bosque aguajal fueron registradas especies raras como Isoetes panamensis y Schizeae poeppigiana, que estan asociados a otras especies de helechos y otras familias herbáceas.

En consecuencia, la distribución de especies terrestres y hemiepífitas son las que mejor indican la preferencia de un tipo de bosque, mientras las especies epífitas como Elaphoglossum latifolium, pueden distribuirse en 
-cualquiera de estos tipos de bosques, donde encuentre mejores condiciones para su establecimiento y desarrollo.

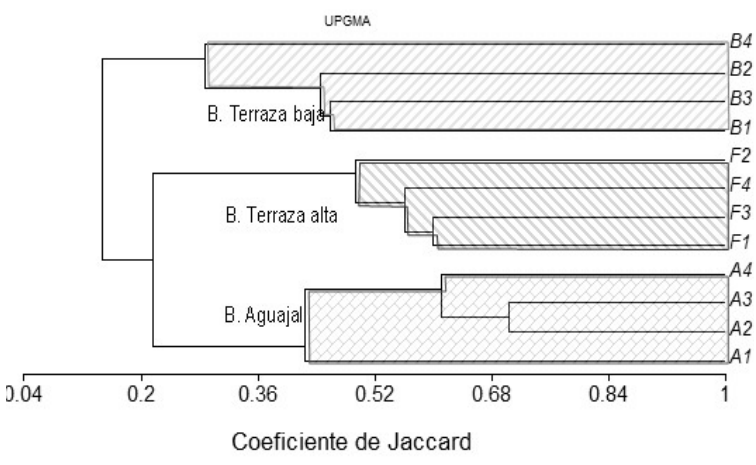

Figura 3. Análisis de agrupamiento de especies en transectos mediante el coeficiente de Jaccard, en tres formaciones vegetales en el Sur este de la Amazonia peruana.

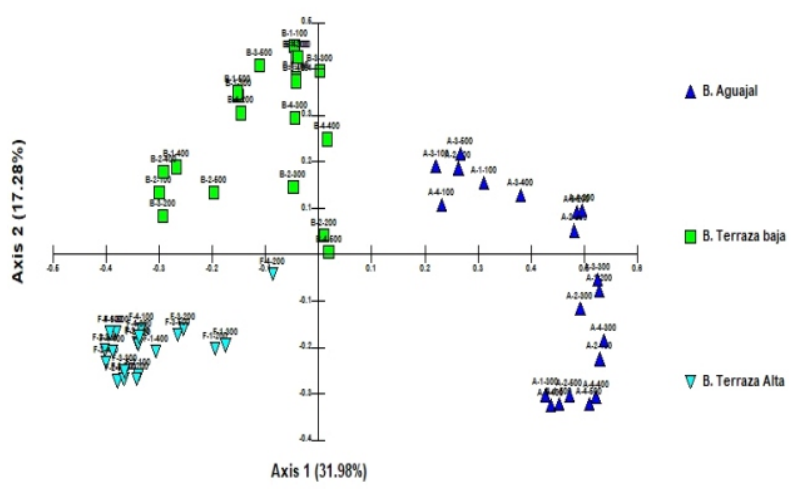

Figura 4. Ordenación de sub parcelas $(5 \mathrm{~m}$. x $100 \mathrm{~m}$.) en Principales Coordenadas para tres formaciones vegetales en el Sur Este de la Amazonia peruana.

\section{Discusión}

En una hectárea (4 transectos de 5m. x 500m.) de bosque de terraza alta (tierra firme) del sector río Los Amigos, se encontró 38 especies de pteridofitas (Tabla 1), esta cantidad es inferior a los documentados para el bosque de tierra firme de Cuyabeno Parque Nacional Yasuní ( Ecuador), Loreto y -
-Madre de Dios (Perú) (Tuomisto \& Poulsen 1996, Tuomisto \& Roukolainen 1994, Ruokolainen et al. 1994, Tuomisto et al. 2002), a excepción de Colombia donde el promedio de especies por transecto es menor al encontrado en el presente estudio (Tuomisto \& Roukolainen 2005).

No obstante, para documentar la flora pteridológica utilizaron diferentes tamaños de parcelas y transectos en los bosques de Cuyabeno I \& II (Ecuador), Área de Investigación Ecológica de Guama y bosques fragmentados (Brasil), y Zona Reservada Allpahuayo - Mishana, Santa Rosa, Huanta, Manití y Nauta (Norte de Perú) (Tuomisto \& Poulsen 1996, Ruokolainen \& Tuomisto 1997, Tuomisto \& Poulsen 2000; Cárdenas et al. 2003, Rodríguez et al. 2004, Barradas \& Prado 2005), donde reportaron diferentes cantidades de especies de pteridofitas, esto limita la comparación con nuestros datos. Sin embargo, el uso de transectos de $5 \mathrm{~m}$. x 500m. evaluados en el sector río Los Amigos, son más efectivos para registrar la riqueza de especies de los bosques del llano amazónico, que en parcelas de 1 hectárea continúa de bosque (Poulsen \& Nielsen 1995, Poulsen et al. 2006, Tuomisto \& Poulsen 1996, 2000).

Localmente, los bosques de colina versus los bosque de terraza alta ambos denominados bosques de tierra firme y están bien diferenciados en situ y a través de imágenes de satélite (Tuomisto et al. 2003 b, c). El bosque de colina por su topografía presenta fisonomía disectadas, donde se genera elevado número micro ambientes húmedos adecuados para el establecimiento de pteridofitas, razón por lo cual estos hábitats presentan alta diversidad en el llano amazónico (Young \& León 1989, Tuomisto et al. 2003 b, Salovaara et al. 2004). 
Al incrementar el esfuerzo de muestreo en ambos tipos de bosques (B. Terraza alta y B. Colinas) tienden a aumentar el registro de especies de pteridofitas (Ruokolainen \& Tuomisto 1994, Tuomisto et al. 2002, Tuomisto \& Ruokolainen 2005).

Del mismo modo, la riqueza de especies de pteridofitas registradas en el bosque inundable es alta para el área de estudio, y es superior a los bosques de Iquitos (Ruokolainen et al. 1994, Cárdenas et al. 2003, Tuomisto et al. 2003 b, Tuomisto \& Ruokolainen 2005) y de Brasil (Rodríguez et al. 2004, Barradas \& Prado 2005). También, las 44 especies de pteridofitos del bosque aguajal es superior al bosque aguajal del Parque Nacional Yasuní (Tuomisto et al. 2003b). Según nuestra revisión literaria, existen pocos estudios realizados en este grupo plantas en bosque aguajal. Esta formación vegetal fue estudiado recientemente en Madre de Dios, donde se documentó su diversidad de especies de flora y fauna, concluyendo que es una de las formaciones vegetales más diversas de la Amazonia sur peruana, siendo los helechos, uno de los grupos de plantas con alta riqueza de especies (Janovec et al. 2013), pero por su difícil accesibilidad no se han intensificado estudios en los bosque aguajales de laAmazonía.

En términos de abundancia, el bosque aguajal presenta la más alta cantidad de individuos de pteridofitas, el cual posee el $63.5 \%$, este es superior al bosque de terraza baja (estacionalmente inundable) y terraza alta (tierra firme) del sector río Los Amigos, a pesar de este atributo no posee la más alta riqueza de especies de la flora pteridológica. La variación de la abundancia y riqueza de especies de pteridofitas dentro del aguajal, se puede- -atribuir a la presencia de sub tipos de vegetación (Janovec et al. 2003). Su abundancia promedio ( $\dot{\mathrm{X}}=2.4$ individuos), es superior a los registrados por Tuomisto et al. (2003b). En contraste, la abundancia de los transectos del bosque inundable ( $\dot{\mathrm{X}}=1.3$ individuos) y bosque de tierra firme $(\dot{X}=7.3$ individuos) son inferiores a los reportes de otros lugares de la Amazonía (Ruokolainen \& Tuomisto 1997, Tuomisto \& Poulsen 2000, Tuomisto et al. 2002, Tuomisto et al. 2003 b, Rodríguez et al. 2004,Tuomisto \& Ruokolainen 2005).

Según el índice de Shannon $\left(H^{\prime}\right)$ el bosque de terraza alta $\left(H^{\prime}: 2.56\right.$ en $\left.1 \mathrm{ha}\right)$ presenta la más alta diversidad de especies, y en menor los bosque aguajal $\left(H^{\prime}: 2.53\right)$ e inundable $\left(H^{\prime}\right.$ : 2.35). Estos resultados no coinciden con el indicador de riqueza de especies, porque este índice se basa en el grado equidad de todas las especies (Moreno 2001). En contraste, el índice de diversidad de Margalef $\left(D_{M_{g}}\right)$ confirma que el bosque inundable $\left(D_{M g}: 6.52\right)$, es el más diverso que los otros dos tipos de bosques evaluados (Tabla 1). Según este índice indica que los bosques de tierra firme e inundable del Área de Investigación Ecológica de Guama (Brasil) son menos diverso especies en flora pteridológica (Rodríguez et al. 2004). Y la diversidad promedio por transecto (índice Shannon-Wiener $H^{\prime}$ ) del bosques de tierra firme en nuestra área de estudio, es inferior a los bosques ubicados en Parque Nacional Yasuní, Cuyabeno (Ecuador) y Nauta (Perú) y superior a los bosques de Manití y Huanta (Perú) (Tuomisto et al. 2003 b, Tuomisto \& Poulsen 2000). También, la diversidad promedio por transecto del bosque inundable y aguajal son inferiores a los reportados por Tuomisto et al. (2003b). En términos generales la diversidad promedio para el bosque de - 
-tierra firme $\left(H^{\prime}: 2.19\right)$ y terraza baja $\left(H^{\prime}\right.$ : 2.0) registrada en nuestra área de estudio, es inferior a los bosques de tierra firme y superior a los bosques inundables de Colombia, Ecuador, Norte y Sur de Perú (Tuomisto \& Roukolainen 2005).

Estas diferencias en diversidad de pteridofitas en los tipos de bosques estudiados, las atribuimos a las condiciones ambientales del sitio (suelo y humedad), mientras que la diversidad beta en bosques tropicales es alta en Panamá (Duivenvoorden et al. 2002), y bajo en los bosques de Perú y Ecuador (Condit et al. 2002), este patrón sugiere por su limitada dispersión y especiación, que influye sobre el reemplazo de especies de árboles.

No obstante, la diversidad beta de pteridofitas entre los tipos de bosques es alta, solo comparten especies entre $24 \%$ y $30 \%$, el cual confirma el alto grado de reemplazamiento de especies (Moreno 2001), mientras que Tuomisto (2010) sugiere que el número de especies distintos entre hábitats es la verdadera diversidad beta. En ese sentido, podemos comparar entre el bosque aguajal versus tierra firme $\left(I_{j}: 0.24\right)$, bosque aguajal versus inundable $\left(I_{j}: 0.28\right)$ y bosque tierra firme versus inundable $\left(I_{J}: 0.30\right)$ suman especies 66, 79 y 73 especies de pteridofitas, compartiendo 16, 22 y 22 especies respectivamente. Por su particularidad el bosque inundable cuenta $29 \%$ especies exclusivas, disminuyendo este atributo en el bosque aguajal (20\%) y tierra firme (17\%). Estas diferencias es similar al comparar entre el bosques de terraza y varillal (Cárdenas et al. 2003).

Al analizar la diversidad beta entre transectos (Figura 3), es intermedia dentro del bosque tierra firme (44\% a 57\%), sin embargo- -grado de reemplazamiento es menor entre los bosques de Manití y Huanta (72\%) y mayor en Nauta, Manití y Huanta (<30\%) (Tuomisto \& Poulsen 1996). No obstante, en el bosque aguajal la similaridad en la composición de especies entre transectos es alta oscila entre $41 \%$ a $70 \%$, y entre los dos tipos de bosques tienen baja similaridad (24\% y 28\%), este patrón coincide con lo reportado porTuomisto \& Ruokolainen (2005). Estos patrones fueron encontrados en otros sitios del norte Amazonia peruana (Ruokolainen et al. 1994; Tuomisto et al. 2003c; Tuomisto \& Roukolainen 2005). Por otro lado, en nuestra área de estudio encontramos 13 de 93 especies de pteridofitas, esta cantidad es similar a lo reportado para los bosques de tierra firme, donde 14 de las 40 especies están presentes en Colombia, Ecuador, Norte y Sur de Perú (Tuomisto \& Roukolainen 2005).

Mediante el Análisis de Principales Coordenadas organiza las subparcelas $\left(500 \mathrm{~m}^{2}\right)$ del bosque aguajal, donde muestra presenta un cambio gradual en la composición de especies de pteridofitas, estos cambios se atribuye a los cambios de vegetación dentro del hábitats (Janovec et al. 2013). Mientras que la mayoría de subparcelas del bosque de tierra firme presenta un patrón de alta similaridad (Salovaara et al. 2004; Ruokolainen et al. 1997; Tuomisto \& Poulsen 2000), razón por el cual están muy cercanas entre sí (Figura 4). En contraste las subparcelas del bosque inundable por su similaridad intermedia, se muestran más dispersas que las demás subparcelas del bosque de tierra firme y aguajal. A pesar de la baja similaridad de especies muestran tendencia a agruparse entre sí (p. e. Salovaara et al.2004), y al mismo tiempo parte de las subparcelas tienden a agruparse a las del bosque de tierra 
-firme y en menor cantidad al bosque aguajal. Similares patrones de composición de especies fueron encontrados en los bosques de Colombia, Ecuador, Norte y Sur de Perú (Tuomisto \& Poulsen 1996 y 2000, Tuomisto et al. 2003 c, b, Tuomisto \& Ruokolainen 2005, Cárdenas et al. 2007, Zuquim et al. 2009).

\section{Conclusiones}

El bosque inundable de sector Los Amigos, presenta la más alta riqueza y diversidad, seguidos por los bosques de Aguajal y Terraza alta. No obstante, el bosque aguajal presenta la más alta abundancia en individuos de Pteridofitas. Es baja la similitud de especies de Pteridofitas, que comparten entre tipos de bosques, solo 13 especies se distribuyen en los 3 tipos de bosques. Se confirma que las pteridofitas están restringidas por las condiciones ecológicas de cada tipo de vegetación.

\section{Agradecimientos}

Instituciones: Universidad Nacional de San Antonio Abad del Cusco (UNSAAC), Asociación para la Conservación de la Cuenca Amazónica (ACCA) y Instituto de Investigación Botánica deTexas (BRIT). Colaboradores: Nigel Pitman, John Janovec, Luis Chambi, Gabriel Alarcón. Por sus identificaciones Taxonómicas: Blanca León, Robbin Moran y Patricia Álvarez.

\section{Literatura citada}

Araujo, M. B., 1999. Distribution Patterns of Biodiversity and the Design of a Representative Reserve Network in Portugal. Diversity and Distributions, 5(4): 151-163.

Barradas, M. L. \& Prado, J. 2005. Effects of forest fragmentation on pteridophyte diversity in a tropical rain forest in Brazil. Plant Ecology, 180:87-104

Beukemaa, H. \& van Noordwijk, M. 2004. Terrestrial pteridophytes as indicators of a forestlike environment in rubber production systems in the lowlands of Jambi, Sumatra. Agriculture, Ecosystems and Environment 104: 63-73
Cárdenas, G., Halme, K. J. \& Tuomisto, H. 2007. Riquiza y Diversidad Ecologica de Especies de Pteridofitas en la Zona del Río Yavarí-Mirín, Amazonia Peruana. Biotropica 39(5): 637-646

Cárdenas, G., Aramburu, C., Castro, L., García A., Zegarra, A. \& Flores, M. 2003. Evaluación de pteridofitas en bosques de la zona reservada Allpahuayo - Mishana y Santa Rosa, Loreto. IIAP. Folia Amazónica 14 (1): 111-115

Cassá de pazos, L. A., Vidoz, F. F., Giudice, G. E., Ramos J.P, Luna M.I. \& De la Sota E.R. 2010. Diversidad de helechos y licofitas del Parque Nacional Lago Puelo (Chubut-Argentina). Boletín de la Sociedad Argentina de Botánica 45 (3-4): 383 403.

Condit, R., Pitman, N., Leigh, E. G. Jr., Chave, J., Terborgh, J., Foster, R.B., Nuñez P., Aguilar, S., Valencia, R., Villa, G., Muller-Landau, H.C., Losos, E. \& Hubbell, S. P. 2002. BetaDiversity in Tropical Forest Trees. Science Vol. 295:666-669

Duivenvoorden, J. F., Svenning, J.C. \&Wright., S. J. 2002. Beta Diversity in Tropical Forests. Science's Compass.Vol. 295: 636-637.

Encarnación, F., Zarate, R. \& Ahuite, M. 2008. Temática Vegetación en; Zonificación Ecológica y Económica del departamento de Madre de Dios. Convenio GOREMAD y IIAP, $74 \mathrm{p}$.

García, R. 2005. Linking floristic patterns to edaphic gradients and remote sensing in Peruvian Amazonia. Tesis de Maestría. Universidad de Missouri - Saint Louis, $95 \mathrm{p}$.

Gómez, J. A. 2012. Estudio de los efectos ambientales sobre la morfofisiología de algunos helechos en diferentes ambientes. Reporte para obtener el diploma de esta especialización en la Universidad Veracruzana, 141p.

Granville, J. J. 1984. Monocotyledons and pteridophytes indicators of environmental constraints in the tropical vegetation. Candollea 39: 265-269

Holdridge L. 1978. Ecología basada en zonas de vida. Centro Científico Tropical, IICA. San José.

Householder, H. 2007. Diversity, natural history, and conservation of vanilla of Madre de Dios, Perú. Master's thesis. Environmental Sciences Program of Texas Christian University. U.S.A. 1-103.

James E.Watkins, J. R. \& Cardelús, C. 2009. Habitat Differentiation of Ferns in a Lowland TropicalRain Forest. Colgate University, Department of Biology. American Fern Journal 99(3):162-175 
Janovec, J., Householder, E., Tobler, M., Valega, R., Von May, R., Araujo, J., Zelski, S., Shearer, C., Jiménez, M., Wells. J., Chambi, B., Herrera. F. \& Perez, M. 2013. Evaluación de los actuales impactos y amenazas inminentes en aguajales y cochas de Madre de Dios, Perú. WWF, Lima, Perú, 244 p.

Janusz, E. 2002. Floristics and hydrology of upper Amazonian swamps, Madre de Dios, Peru. Master's thesis. Departamento de Biologia de Wake Forest University. Winston-Salem, North Carolina. U.S.A., 2-66.

Kalliola, R., M. Puhakka, J. Salo, H. Tuomisto, \& Ruoklainen, K. 1991a. The dynamics, distribution and classification of swamp vegetation in Peruvian Amazonia. Annales Botanici Fennici. 28: 225-239.

Kessler M. 2002. Range size and its ecological correlates among the pteridophytes of Carrasco National Park, Bolivia. Global Ecology \& Biogeography.Vol. 11, 89-102

Kovach Computing Services, 2007, Multi_Variate Statistica Package v 3.13, Pentraeth, Wales, U.K.

Martínez, O. G. \& Prado, D. E. 2013. Distribución fitogeográfica de helechos y licófitas en el valle de lerma (salta - argentina). Chloris chilensis, Revista chilena de flora y vegetación. Año 16, $\mathrm{N}^{\circ} 2$. URL: http:www.chlorischile.cl

Mazer, S. 1997. Floristic composition, soil quality, litter accumulation, and decomposition in terra firme and floodplain bosques near Pakitza, Peru: Pág. 89-126. En: D. E.Wilson and A. Sandoval (eds.), Manu: The biodiversity of southeastern Peru. Smithsonian Institution and Editorial Horizonte, Lima.

Mondragón, A. \& García, J. A. 2011. Pteridoflora (polypodiophyta) de una parcela en el bosque ribereño "El Tambor" del Municipio Morán, Estado Lara, Venezuela. Pittieria 35 (2011): 95-108

Moreno, C. 2001. Métodos para medir la biodiversidad. M\&T - Manuales y Tesis SEA, vol.1. Zaragoza, 84 pp.

Pitman, N. 2000. A large-scale inventory of two Amazonian tree communities. Dissertation. Master's thesis. Department of Botany, Duke University, Durham, U.S.A.

Poulsen, A. D. \& Nielsen, I. H. 1995. How many ferns there in one hectare of tropical rain forest?. American Fern Journal 85 (1): 29-35
Poulsen, A. D., Tuomisto, H. \& Balslev, H. 2006. Edaphic and Floristic Variation within a 1-ha Plot of Lowland Amazonian Rain Forest. Biotropica 38(4): 468-478.

Rodrigues, S.T., de Almeida, S. S., Andrade, L. C., Barros, I. C. \& Van der Berg, M. E. 2004. Composição florística e abundância de pteridófitas em três ambientes da bacia do rio Guamá, Belém, Pará, Brasil. Acta Amazónica.VOL. 34(1): 35 - 42

Rodríguez, L., Pacheco, L. \& Zavala, J. A. 2008. Pteridofitas indicadoras de alteración ambiental en el bosque templado de San Jerónimo Amanalco, Texcoco, México. Rev. Biol. Trop. (Int. J.Trop. Biol. ISSN-0034-7744)Vol. 56 (2): 641-656

Ruokolainen, K., Linna, A. \& Tuomisto, H. 1997. Use of Melastomataceae and Pteridophytes for revealing phytogreographical patterns in Amazonian rain forest. Journal of Tropical Ecology, 13: 243-256.

Ruokolainen, K., Tuomisto, H., Ríos, R., Torres, A. \& García, M. 1994. Comparacion florística de doce parcelas en bosque de tierra firme en la Amazonia Peruana. Acta Amazonica, 24 (1/2): 31 48.

Salovaara, K. J., Cárdenas G. G. \& Tuomisto, H. 2004. Forest classification in an Amazonian rainforest landscape using pteridophytes as indicator species. Ecography 27:689-700.

Soria-Auza, R. W. 2009. Diversity and Biogeography of Ferns and Birds in Bolivia: Applications of GIS Based Modelling Approaches. Erlangung des Doktorgrades, Universität Göttingen. 141 pp.

Santiago A. C., Barros I. C. \& Sylvestre L. 2004. Pteridófitas ocorrentes em três fragmentos florestais de um brejo de altitude (Bonito, Pernambuco, Brasil). Acta bot. bras. 18(4): 781792.

Tryon, R. M. \& Stolze, R. G. 1989. Pteridophyta of Peru. Part I. 1. Ophioglossaceae - 12. Cyatheaceae. Fieldiana Botany, New Series 20:3- 145.

Tryon, R. M. \& Stolze, R. G. 1991a. Pteridophyta of Peru, Part IV. 17. Dryopteridaceae. Fieldiana Botany, New Series 20:3-176.

Tryon, R. M. \& Stolze, R. G. 1991b. Pteridophyta of Peru, Part V. 18. Aspleniaceae - 21. Polypodiaceae. Fieldiana Botany, New Series 32:3-190

Tryon, R. M. \& Stolze, R. G. 1994. Pteridophyta of Peru. Part. VI. 22. Marsileaceae - 28. Isoëtaceae. Fieldiana, Botany 34:1-123. 
Tuomisto, H. \& Poulsen, D. 1996. Influence of edaphic specialization on pteridophyte distribution in neotropical rain forests. Journal of Biogeography Vol. 23: 283-293.

Tuomisto, H. \& Ruokolainen, K. 1994 . Distribution of Pteridophyta and Melastomataceae along an edaphic gradient in an Amazonian rain forest. Journal of Vegetation Science 5: 25-34

Tuomisto, H. \& Ruokolainen, K. 2005. Environmental heterogeneity and the diversity of pteridophytes and Melastomataceae in western Amazonia. Biol. Skr. 55:37-56.

Tuomisto, H. \& Ruokolainen, K. 1997. The role of ecological knowledge in explaining biogeography and biodiversity in Amazonia. Biodiversity and Conservatión 6: 347-357

Tuomisto, H. 1998 (a). What Satellite Imagery and Large-Scale Field Studies Can Tell About Biodiversity Patterns in Amazonian Forests. Annals of the Missouri Botanical Garden, Vol. 85, No. 1, pp. 48-62

Tuomisto, H. 2010. A diversity of beta diversities: straightening up a concept gone awry. Part 1. Defining beta diversity as a function of alpha and gamma diversity. Ecography 33: 2-22

Tuomisto, H., Poulsen, A.D., Ruokolainen, K., Moran, R. C., Quintana, C., Celi, J. \& Cañas, G. 2003 (b). Linking floristic patterns with soil heterogeneity and satellite imagery in Ecuadorian Amazonia. Ecological Applications, 13(2): 352-371

Tuomisto, H., Ruokolainen, K., Aguilar, M. \& Sarmiento, A. 2003 (c). Floristic patterns along a 43- km long transect in an Amazonian rain forest. Journal of Ecology. 91: 743-756

Tuomisto, H., Ruokolainen, K., Poulsen, A.D., Moran, R.C., Quintana, C., Cañas, G. \& Celi, J. 2002. Distribution and Diversity of Pteridophytes and Melastomataceae along Edaphic Gradients in Yasunı' National Park, Ecuadorian Amazonia. BIOTROPICA 34(4): 516-533

Tuomisto, H. \& Poulsen, A. D. 2000. Pteridophyte diversity and species composition in four Amazonian rain forests. Journal of Vegetation Science 11: 383 396.

Tuomisto, H., Poulsen A.D. \& Moran, R.C. 1998. Edaphic distribution of some species of the fern genus Adiantum Western Amazonia. Biotropica, Vol. 30, $\mathrm{N}^{\circ}$ 3. Pp. 392-399.
Williams-Linera, G., Palacios-Ríos, M. \& Hernández-Gómez, R. 2005. Fern richness, tree species surrogacy, and fragment complementarity in a Mexican tropical montane cloud forest. Biodiversity and Conservation 14: 119-133

Young, B. 2007. Distribucion de las especies endemicas en la vertiente oriental de los Andes en Peru y Bolivia. NatureServe, Arlington, Virginia, EE UU. $90 \mathrm{p}$.

Young, K. R. \& León B. 1989. Pteridophyte species diversity Central Peruvian: Importance edaphic specialization. BrittoniaVol.41, No. 4. 389-395.

Yusuf, F. B., Tan, B. C. \& Turner, I. M. 2003. what is the minimum area needed to estimate the biodiversity of pteridophytes in naturaland manmade lowland forests in malaysia and singapore? Fern Gaz. 17(1): 1-9.

Zuquim, G. 2006. Diversidade beta da comunidade de pteridófitas de florestas de terra firme na Amazônia central. Instituto Nacional de Pesquisas da Amazônia. 61 p. 